\title{
Synthesis, Construction, and Validation of a Fractal Surface
}

\author{
Giuseppe Ruello, Member, IEEE, Pablo Blanco-Sánchez, Antonio Iodice, Senior Member, IEEE, \\ Jordi J. Mallorqui, Member, IEEE, Daniele Riccio, Senior Member, IEEE, Antoni Broquetas, Member, IEEE, and \\ Giorgio Franceschetti, Life Fellow, IEEE
}

\begin{abstract}
Fractal geometry provides reliable models to describe geometrical properties of natural surfaces. Therefore, their use in the electromagnetic scattering methods deserves careful research. In order to have complete insight into the phenomenon, a measurement campaign on a fractal surface in a controlled environment is a key step. In this paper, we propose a technique for building a fractal surface that can be used for electromagnetic scattering evaluation purposes. The surface characteristics are imposed by computer synthesizing a bandlimited Weierstrass-Mandelbrot function, whose actual shape is constructed by means of a cheap innovative technique: the synthesized surface is made from cardboard covered with aluminum foil, which gives a conducting surface and creates the micro-scale conditions, useful to represent manufacturing errors. Statistics of the overall surface shape are then measured, analyzed and compared with the imposed ones, providing and verifying the rationale for a fully controlled surface to be applied in any kind of experiment on natural surfaces.
\end{abstract}

Index Terms-Electromagnetic scattering by rough surfaces, fractals.

\section{INTRODUCTION}

$\mathbf{T}$ ODAY it is widely known that Nature follows fractal laws on wide ranges of scales. Hence, scientists who study or try to describe natural phenomena have to consider the use of fractal geometry. From the theory of chaos to land surface description, from sea surface synthesis to stock market analysis, fractal concepts are used in more and more research fields [1]-[9]. In particular, it is by now widely recognized (see, e.g., [1]-[3], [20], and [26]) that fractal models are very useful in the description of natural surfaces because they properly account for the scale invariance property typical of such surfaces. In addition, as Mandelbrot argued [1], classical parameters usually employed to describe natural surfaces (i.e., standard deviation and correlation length) change when the scale at which the surface

Manuscript received February 24, 2005; revised November 22, 2005. The work of G. Ruello was supported by the Spanish MEC Grant SB2003-0290. This work was supported by the Spanish MCYT and FEDER funds under project TIC2002-04451-C02-01, by the Spanish-Italian Integrated Action HI2004-0076/IT2390, and by the Campania Region "Centro Regionale di Competenza su Analisi e Monitoraggio del Rischio Ambientale" and "Centro Regionale di Competenza su Tecnologie dell'Informazione e della Comunicazione."

G. Ruello, A. Iodice, D. Riccio, and G. Franceschetti are with the Dipartimento di Ingegneria Elettronica e delle Telecomunicazioni, Università di Napoli "Federico II," 80125 Napoli, Italy (e-mail: ruello@unina.it; iodice@unina.it; Daniele.Riccio@unina.it; gfrance@unina.it).

P. Blanco-Sánchez, J. J. Mallorqui, and A. Broquetas are with the Department of Signal Theory and Communications, Universitat Politecnica de Catalunya, Campus Nord UPC-D3, 08034 Barcelona, Spain (e-mail: pblanco@ tsc.upc.edu; mallorqui@tsc.upc.edu; toni@ tsc.upc.edu).

Digital Object Identifier 10.1109/TGRS.2006.870433 is observed changes. Conversely, fractal parameters of a natural surface are independent of the observation scale.

The most useful fractal model for natural surfaces is the fractional Brownian motion (fBm) [7], [8]. It is a stochastic nonstationary process described in terms of the probability function of its increments, whose sample functions are everywhere continuous, but everywhere nondifferentiable. This process is conveniently approximated by the Weierstrass-Mandelbrot (WM) fractal function, which is a frequency sampled version of an $\mathrm{fBm}$, and provides an analytical expression of the surface shape as a superposition of sinusoidal tones [14].

In view of the considerations reported above, it seems reasonable to expect that use of fractal models in the computation of scattering from natural surfaces leads to results that correspond better to the measurements. However, this has not yet been convincingly demonstrated and is still an open point of discussion: therefore, it is of crucial importance to devise reliable methods to verify the validity of fractal scattering models. In fact, the use of the $\mathrm{fBm}$ geometrical model in conjunction with electromagnetic scattering models like the Kirchhoff approximation (KA) and the small perturbation method (SPM) has been implemented in [9] and [13] and allows an evaluation of the second-order statistics of the electromagnetic field scattered from natural surfaces (i.e., the mean scattered power). The use of the WM in the KA, as well as in the extended boundary condition method (EBCM), has also been presented in [15] and [16] and gives the analytical evaluation of the field scattered by a single surface realization and the scattered power, via Monte Carlo simulations. However, up to now, for both the fBm and the WM, the comparison between scattering method results and the measured data has been done by using scattering data reported in literature, and relative to scattering surfaces whose fractal parameters were not known, so that a best fit procedure was needed to estimate them [9].

Accordingly, the need for a measurement campaign devoted to the study of the scattering of electromagnetic fields from fractal surfaces in a controlled environment (i.e., with known fractal and electromagnetic parameters) is now strongly felt by the remote sensing community. So far, to the best of the authors' knowledge, such a measurement campaign has not been carried out. In fact, previous measurements were performed on Gaussian classical surfaces [17]-[19].

In this paper, we present an innovative approach to design and build a fractal surface. We present the rationale from which the synthesis, the construction and the validation of the surface were derived, along with its implementation in an actual case. These topics are discussed according to the following organization. 
In Section II, the rationale which defines the design choices is presented. The main constraints are identified and a solution to the limitations they pose is proposed. Identified solutions define a strategy whose implementation guarantees the procedure reproducibility.

The synthesis technique is detailed in Section III. We used a WM function for the surface description. The proposed fractal model is described in terms of few parameters, whose values are chosen in terms of the scattering field evaluation requirements.

In Section IV, the procedure for building the fractal surface is described. The construction of the surface was determined by the contour plot representation of a WM function. Cardboard slices were shaped in accordance with the numerically evaluated contour curves, and superimposed to obtain a long-scale cardboard topography. Then, high-frequency roughness was added as pieces of wrinkled aluminum foil, so that the surface would have perfectly reflecting properties.

Then, we verified that this surface had the required properties via an optical analysis. In Section V, we discuss how we used the white light triangulation method to precisely measure a digital elevation map of the synthesized surface. Measurement accuracy and precision were chosen so that we could retrieve reliable values of the fractal parameters.

In Section VI we employ both spatial and frequency domain analysis techniques to preliminarily verify that the synthesized surface is fractal and then to retrieve its fractal parameters. Obtained results prove that the estimated fractal parameters are consistent with the imposed ones.

Section VII is devoted to the final discussion and to the presentation of new ideas to exploit the synthesized surface.

\section{CONSTRAints AND OPERATION PROTOCOL}

The design of a fractal surface is limited by several constraints. Experiment aims, synthesis procedure, work environment, budget availability, measurement repeatability, and so on, pose several restrictions. In this section, we identify the main requirements that regulate the surface construction and we propose an operational procedure the implementation of which guarantees the reproducibility of the process.

\section{A. Work Environment}

Even if an external campaign has the evident advantage of working on actual surfaces, it presents several drawbacks related to the scarcely controllable work environment and the campaign costs. Therefore, a very complicated calibration procedure is required and the economical costs of such a procedure can be extremely high. Conversely, a laboratory campaign guarantees stable measurement conditions, providing a high degree of measurement repeatability and reliability at low cost.

In this paper, we present the construction of a surface for a measurement campaign in a controlled environment. All the problems related to this kind of approach are discussed in the following paragraphs.

\section{B. Surface Nature}

Once the choice of a laboratory measurement campaign has been made, the need to decide whether to analyze a natural or an artificial surface arises. The first option is, in principle, more attractive, but it poses problems related to the transportation and handling of an actual surface in an anechoic chamber. In addition, an expensive evaluation of the surface dielectric properties would be required in order to fully characterize the electromagnetic scattering behavior.

Conversely, the use of an artificial surface allows tailoring the occupation of space, the surface weight and dimensions, its dielectric properties in accordance with laboratory constraints and experiment aims. Hence, it can be an attractive solution, if an appropriate surface synthesis is performed and if cheap, light construction materials are chosen.

Here we adopted the second approach.

\section{Synthesis Requirements}

The construction of a controlled artificial surface has to be governed by a reliable analytical expression, in accordance with laboratory dimensions and experiment goals.

Fractal geometry proved to be a versatile and appropriate instrument for surface description, as presented in Section III. Constraints on the surface parameters (height and resolution precision, dimensions, and so on) are imposed by the working conditions as well as by the electromagnetic scattering theory requirements.

The surface size must be set with reference to conflicting requirements: it has to be limited by the anechoic chamber size; on the other hand, a significant scattered field contribution has to be measured and the edge effect has to be limited; therefore, the surface linear size must be much larger than the considered electromagnetic wavelength. Our choices are detailed in Section III.

\section{Surface Materials}

As far as the surface materials are concerned, they have to guarantee easy construction, limited weight and sufficient rigidity. In this paper, we present an experimental work on a cardboard-aluminum surface. Cardboard is very light and the surface can be shaped with a high degree of precision with scales from the longest to those of fractions of a centimeter. With aluminum we can create roughness on the smallest scales (just a few millimeters), and thus the surface becomes a perfectly conducting material. This avoids the problem of estimating the surface dielectric properties. Quantitative details on the surface construction are provided in Section IV.

\section{E. Validation Instruments}

Once the surface has been created, it is crucial to validate its statistical characteristics. First of all, the measurement technique should not change the surface shape and roughness, so optical measurements are preferred to contact ones; in addition, the performances of the instruments have to fulfil the application requirements.

When using the constructed surface in electromagnetic scattering experiments, the incident field wavelength $\lambda$ is the reference yardstick. In order to achieve an accurate description of the surface, all scales that play a significant role in the electromagnetic scattering phenomenon have to be measured. Therefore, a height precision of the order of at least $\lambda / 20$ is required. As for spatial resolution, $\lambda / 10$ pixel spacing provides accurate information on all scales of interest. Typical performances 
of commercial optical instruments fulfil such a requirement in the microwave frequency regime. Details of the technical specifications of surface measurement instruments are provided in Section V.

The concepts presented here define a procedure of synthesizing, constructing and validating a fractal surface. In the following sections, the solutions we adopted to implement the presented protocol are discussed in detail.

\section{Fractal SURfaces: CONCEPTS AND SYNTHESIS}

The $\mathrm{fBm}$ is an always continuous, but always nondifferentiable process, described in terms of its increment pdf [7], [8]. A stochastic process $z(x, y)$ is an $\mathrm{fBm}$ surface if, for every $x, y, x^{\prime}, y^{\prime}$, it satisfies the following relationship:

$$
\begin{aligned}
\operatorname{Pr}\left\{z(x, y)-z\left(x^{\prime}, y^{\prime}\right)<\bar{\zeta}\right\} & \\
= & \frac{1}{\sqrt{2 \pi} s \tau^{H}} \int_{-\infty}^{\bar{\zeta}} \exp \left(-\frac{\zeta^{2}}{2 s^{2} \tau^{2 H}}\right) d \zeta
\end{aligned}
$$

where $\tau$ is the distance between the points $(x, y)$ and $\left(x^{\prime}, y^{\prime}\right)$, Pr stands for "probability" and the two parameters that control the $\mathrm{fBm}$ behavior are as folllows:

- $H$ : the Hurst coefficient $(0<H<1)$, related to the fractal dimension $D$ by means of the relationship $D=$ $3-H$

- $s$ : the standard deviation, measured in $\left[\mathrm{m}^{(1-H)}\right]$, of surface increments at unitary distance, a real parameter related to an $\mathrm{fBm}$ characteristic length, the topothesy $T$, by means of the relationship $s=T^{(1-H)}$.

It has been demonstrated [8], [20], [27] that the spectrum $S(k)$ of an isotropic $\mathrm{fBm}$ process is a power law

$$
W(k)=S_{o} k^{-\alpha}
$$

wherein the spectral and spatial domain parameters are related [9] by the following relationships:

$$
\begin{aligned}
\alpha & =2+2 H=8-2-D \\
S_{0} & =s^{2} 2^{2 H} 2 \pi H \frac{\Gamma(1+H)}{\Gamma(1-H)}
\end{aligned}
$$

$\Gamma(\cdot)$ being the Gamma function. Note that from the inequalities $0<H<1$ we get $2<\alpha<4$, which defines the range of allowed values for the spectral slope $\alpha$.

Several techniques have been developed in order to synthesize samples of an $\mathrm{fBm}$ process [1]-[3], [14], following approaches that differ according to the applications considered. In this paper we are interested in a surface description that well approximates the $\mathrm{fBm}$ by employing the minimum number of parameters, related to the physical properties of the surface; thus the characteristics of the synthesized surface to be used in electromagnetic scattering measurements are easily controlled. Within this framework, the WM function is the most appropriate one [1]-[3], [14]. It provides an analytical expression of the surface $z(x, y)$, as follows:

$$
\begin{aligned}
z(x, y)=B \sum_{p=-\infty}^{\infty} C_{p} \nu^{-H p} \\
\\
\quad \times \sin \left[k_{0} \nu^{p}\left(x \cos \Psi_{p}+y \sin \Psi_{p}\right)+\Phi_{p}\right]
\end{aligned}
$$

where $C^{p}, \Phi^{p}, \Psi^{p}$, are random amplitude, phase and direction coefficients; in this paper, $C_{p}$ are chosen to be unitary (hence the amplitude coefficient matches the $\mathrm{fBm}$ spectral law in deterministic sense), $\Phi_{p}$ and $\Psi_{p}$ are uniformly distributed in $[0,2 \pi]$. The four parameters that control the WM behavior are as follows:

- $H$ : the Hurst coefficient $(0<H<1)$, related to the fractal dimension $D$ by means of the relationship $D=$ $3-H$

- $B$ : the vertical overall height scaling factor;

- $\nu$ : the irrational parameter $(\nu>1)$ accounting for the spatial wavenumber spacing between tones;

- $k_{0}$ : the fundamental wavenumber.

Therefore, the WM is a superposition of sinusoidal tones spaced by the irrational parameter $\nu$, hence it is an almost periodic function [11].

The WM function can be seen as the representation of an $\mathrm{fBm}$ process with the same fractal dimension sampled in the wavenumber domain at the discrete spatial wavenumbers $\kappa_{p}=$ $\kappa_{0} \nu^{p}$ [2], [9]. Therefore, for $\nu$ values close to 1 , the WM tones are very crowded and the function well approximates the fBm process [2], [14]. In addition, the spectral parameter $S_{0}$ is related to the WM parameters by the following relationship [9]:

$$
B^{2}=\frac{S_{0}}{2 \pi H} k_{0}^{-2 H}\left(\nu^{H}-\nu^{-H}\right) .
$$

A further modification of this function must be done, by considering that the natural surfaces are fractal in a wide but limited range of scales [1], [3]; hence, the number of tones in our process cannot be unlimited. The truncation criterion depends on the application for which the surface is designed. Therefore, only those tones that significantly contribute to the electromagnetic scattered field formation are of interest in our research: accordingly, we can neglect tones with periods lower than a fraction of the electromagnetic wavelength and much higher than the illuminated surface dimensions. If $X$ and $Y$ are the linear dimensions of the illuminated surface, the number of tones $P$ can be evaluated as [9]

$$
P=\left\lceil\frac{\ln \left(\sqrt{X^{2}+Y^{2}} /(\chi \cdot \lambda)\right)}{\ln \nu}\right\rceil+1
$$

where $\lambda$ is the electromagnetic wavelength and a safety factor $\chi(0<\chi<1)$ has been introduced. Therefore, the summation index $p$ in (3.5) spans from 0 to $P-1$.

In this work, we want to set up a surface to be measured at different frequencies in the microwave region, hence the choice of the parameters is influenced by the frequency bands to be used for measurements. In particular, surface simulation is optimized for an X-band analysis so that the incident wavelength $\lambda$ is about $3 \mathrm{~cm}$. In addition, the highest scale dimension was set at $1.5 \mathrm{~m}$ (about $50 \lambda$ ), providing a reference for the lowest tone, that is for the $k_{0}$ value. As far as the $H$ value is concerned, in the reported experiment a typical value that falls between those relevant to natural surfaces was chosen $(H=0.7)$ [1], [20], [21]. Once the physical parameters have been fixed, as stated above, the $\nu$ value determines how close the tones of the WM function are distributed in the wavenumber domain thus influencing the quality of the approximation provided by the discrete WM spectrum to the continuous $\mathrm{fBm}$ one. In this experiment we chose a 
TABLE I

SiMUlATED SURFACE PARAMETERS

\begin{tabular}{c|c|c|c|c|c|c|c}
\hline \multicolumn{3}{c|}{ Chosen Parameters } & \multicolumn{4}{c}{ Derived Parameters } \\
\hline$k_{0}\left[\mathrm{~m}^{-1}\right]$ & $B[\mathrm{~m}]$ & $H$ & $v$ & $P$ & $S_{0}\left[\mathrm{~m}^{0.6}\right]$ & $\alpha$ & $s\left[\mathrm{~m}^{0.3}\right]$ \\
\hline 5.71 & 0.011 & 0.7 & $0.5 \mathrm{e}$ & 20 & 0.0014 & 3.4 & 0.063 \\
\hline
\end{tabular}

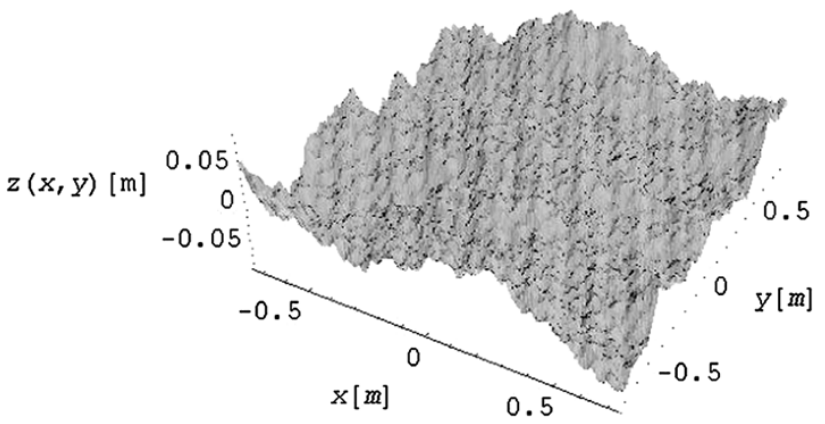

Fig. 1. Realization of a WM function.

$\nu$ value $(\nu=0.5 \mathrm{e}$, e being the Neper number) that would guarantee a good wavenumber sampling of an $\mathrm{fBm}$ process with the same $H$, as confirmed by specialized literature [22].

The chosen values for the four WM parameters $\left(H, B, \nu, k_{0}\right)$ are reported in Table I, as well as the obtained values of the dependent parameters: the number of tones $P$, the spectral amplitude $\mathrm{S}_{0}$, and the spectral coefficient $\alpha, s$. A surface realization of such a process is depicted in Fig. 1 .

\section{Fractal Surface Construction}

In this section, the problems related to the sample surface realization are discussed and the solution adopted is presented, in accordance with the concepts introduced in Section II.

We devised different construction techniques to build the long (from $1.5 \mathrm{~m}$ to $0.5 \mathrm{~cm}$ ) and the short (smaller than $0.5 \mathrm{~cm}$ ) spatial scales. As far as the long scales are concerned, we evaluated the level curves (with an elevation step of $0.5 \mathrm{~cm}$ ) of a natural surface synthesized according to the procedure in Section III by means of an appropriate software code- - see Fig. 2(a). Then, we first built and then superimposed $0.5-\mathrm{cm}$ thick cardboard layers, shaped according to the level curves, obtaining the stepped topography illustrated in Fig. 2(b). The choice of cardboard material allowed setting up a rigid but light structure, easily portable, fulfilling the mechanical constraints identified in Section II. The overall topography dimensions $\left(1.5 \times 1.5 \mathrm{~m}^{2}\right)$ were chosen according to laboratory dimensions and electromagnetic wavelength. In order to reduce the border effect during the electromagnetic scattering measurements, the surface was cut in a circular shape over the horizontal plane, with a $1.5-\mathrm{m}$ diameter.

The next step was to build the spatial scales shorter than $0.5 \mathrm{~cm}$, by putting two layers of randomly wrinkled aluminum foil over the long-scale topography. The advantages of using aluminum are related not only to the low cost and simplicity of this solution, but also to the possibility of considering the surface as a perfectly conducting material in the electromagnetic scattering experiments.

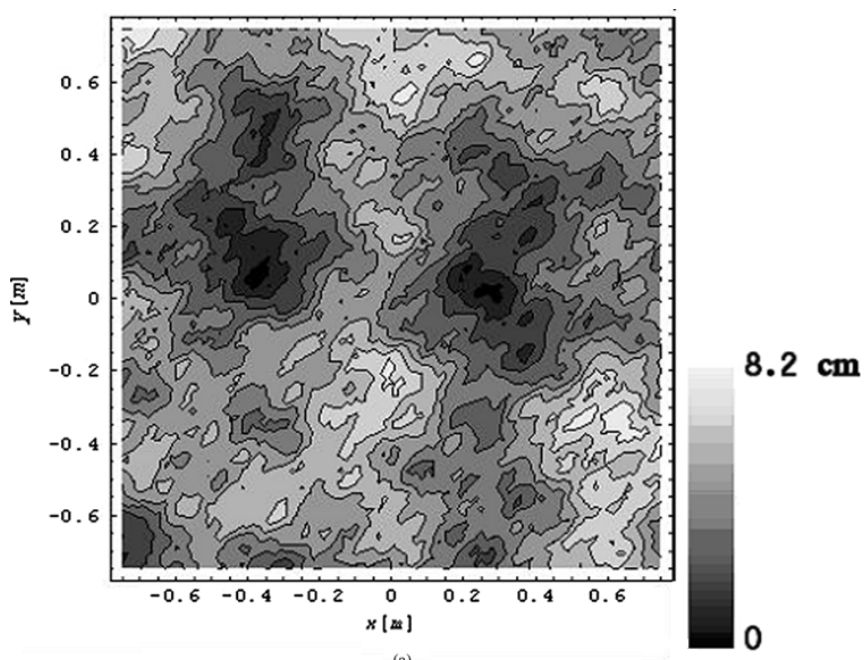

(a)

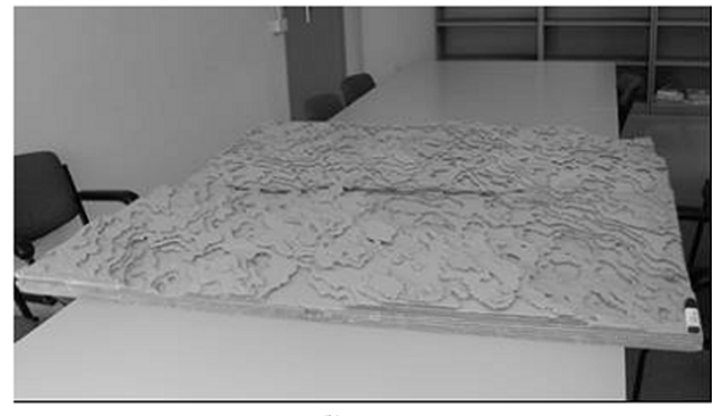

(b)

Fig. 2. (a) Contour plot of the surface depicted in Fig. 1. (b) Long-scale cardboard topography.

We can see the long-scale building as the construction of a sample surface quantized (along the vertical direction) at 0.5 -cm steps. Hence, the long-scale topography includes from the longest to the $0.5-\mathrm{cm}$ spatial scale. The overlaying of the first aluminum layer can be interpreted as an interpolation that adds higher spatial frequency components to the constructed long-scale surface. The correlation between the aluminum layers and the long scales should give the surface a continuum spectrum with an almost linear (in a log-log plane) behavior, as confirmed by the measurements presented in Section VI. The last aluminum layer is responsible for the highest frequencies. In Fig. 3(a), we show a photo of the realized surface from the top, in Fig. 3(b) a close-up of the two aluminum layers, and in Fig. 3(c), a detail of the microscopic roughness.

Such a surface does not lead to complete control of the highfrequency roughness (in our case on the order of some millimeters); this depends on how the aluminum foil is wrinkled. Anyway, at microwave frequencies, it is possible to include all the scales that contribute to the scattered field formation on the long-scale surface. In order to prove our statement, some experiments on the scattering properties of natural surfaces were performed with the method of moments (MoM). In particular, we analyze the effect of a high-frequency random noise on the field scattered by a fractal profile with the same parameters as the constructed surface $\left(H=0.7, s=0.063 \mathrm{~m}^{1-\mathrm{H}}\right)$. The analysis is performed at X-band. A white Gaussian noise with a standard deviation of $0.001 \mathrm{~m}$ was generated and then highpass-filtered with a cutoff frequency corresponding to the inverse of 


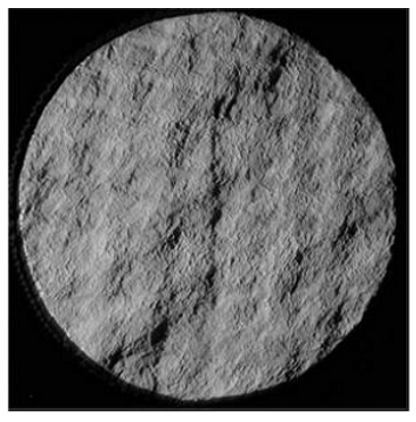

(a)

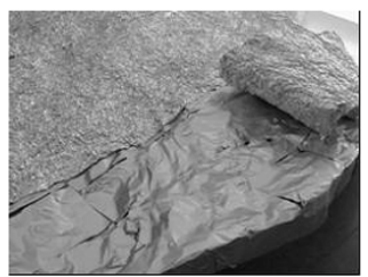

(b)

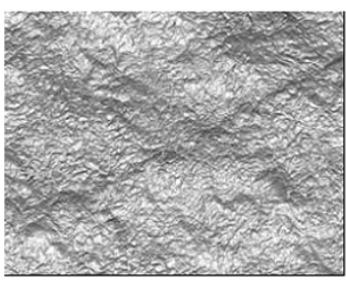

(c)
Fig. 3. (a) Top view of the whole surface. (b) Particular of the low-scale roughness formation. (c) Roughness detail.

$\lambda / 6$. This highpass-filtered noise is then added to the considered profile. The spectrum of the obtained (fractal+noise) profile is shown in Fig. 4(a) (the dotted lines are in correspondence with the spatial frequency relative to $\lambda / 6$ ). In Fig. 4(b) and (c), a $20 \lambda$ portion of the pure fractal and of the noise affected signals is presented. The standard deviation of the difference between these profiles is $0.6 \mathrm{~mm}$, which is one order of magnitude higher than the estimated wrinkling process rms value of $0.07 \mathrm{~mm}$. The field scattered by these profiles was evaluated via the MoM and the comparison is shown in Fig. 5. Note that this is a worst case analysis, because on the constructed surface the aluminum noise is correlated with the long-scale characteristics, therefore the effect on the scattering properties is expected to be lower. In addition, note that the noise power used is overestimated, so that our results will be the same in less propitious conditions, as the case of a smoother surface. It is clearly shown that the presence of this high-frequency noise does not significantly change the scattering properties of the surface.

\section{Fractal Surface Measurement}

Once the fractal surface is constructed, the obtained height profile can be analyzed in order to extract its fractal characteristics. The preliminary step to this validation procedure consists in measuring the height profile of the surface and obtaining its digital model in a raster format. Hence, this section is aimed at describing the height profile measurement step.

The selected optical measurement method is known as structured white light triangulation, which does not need any mechanical global positional equipment and represents an extension of the current light triangulation method typically used to measure distances. Key details of this technique are explained in the following.

The surface is first placed in a fixed position and an optical "camera" based on the structured white light triangulation tech-

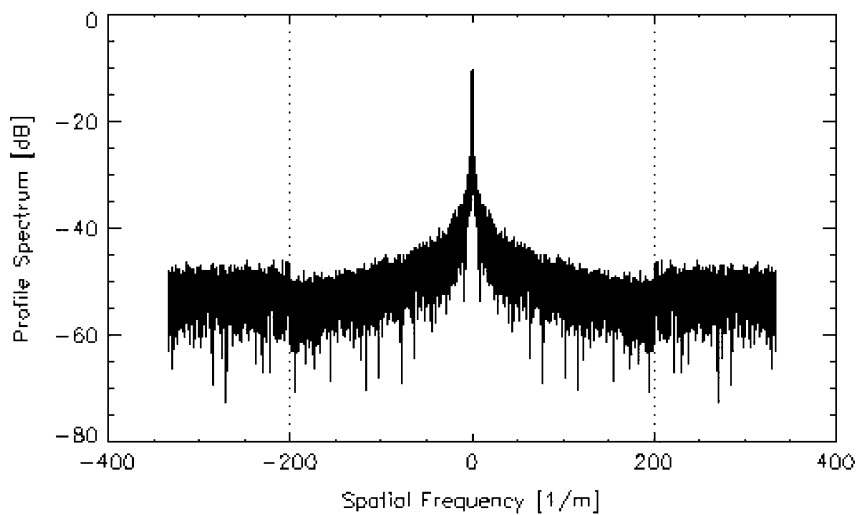

(a)

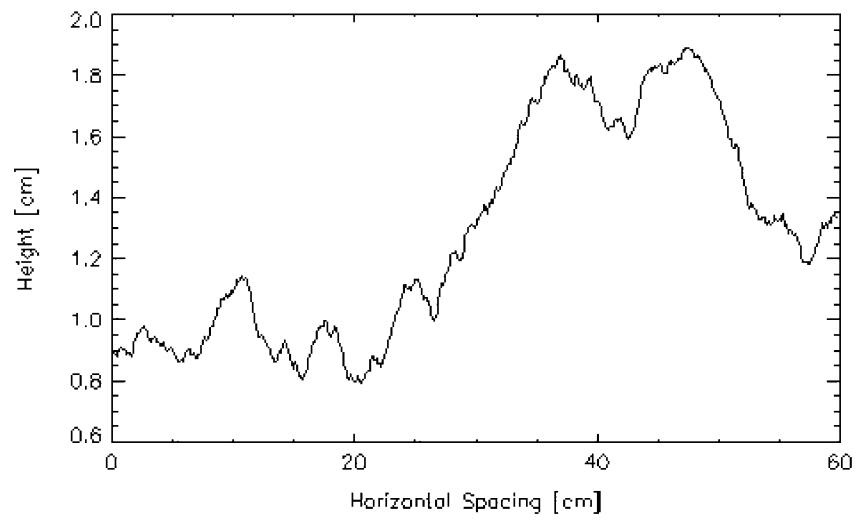

(b)

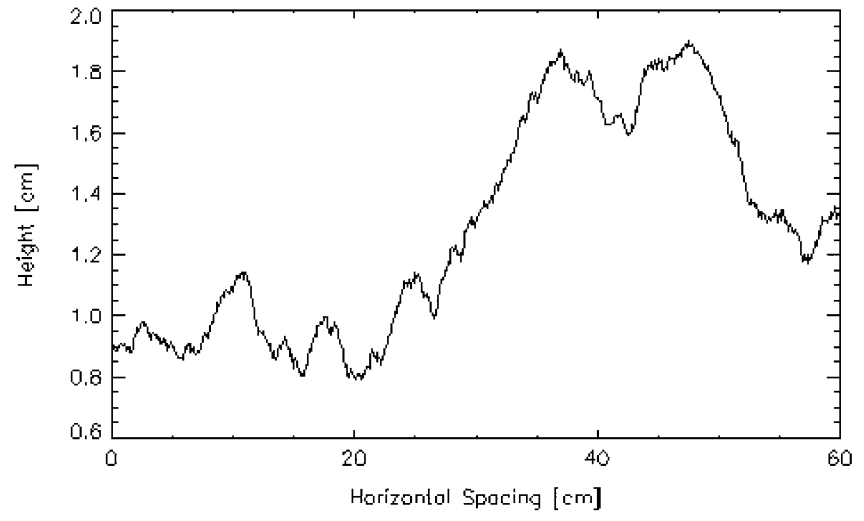

(c)

Fig. 4. (a) Spectrum of the fractal profile with the high-frequency noise (b) $20 \lambda$ portion of the fractal profile. (c) $20 \lambda$ portion of the noisy fractal profile.

nique images the surface. This camera projects a set of vertical, black and white, light fringes on the surface. By means of another camera integrated in the system we observe the surface and the projected fringes. The three-dimensional (3-D) information is obtained by analyzing the deformation suffered by the projected lines, obtaining a set of points of coordinates $x, y, z$ of the fractal surface.

Very high precision of the position measurement is achieved: the obtained maximum separation between measured points (horizontal resolution) is $0.7 \mathrm{~mm}$. This is certainly sufficient, because it is well below the wavelength that will be used in microwave scattering experiments (i.e., $3 \mathrm{~cm}$ at $\mathrm{X}$-band). In addition, the accuracy of height measurements (vertical resolu- 


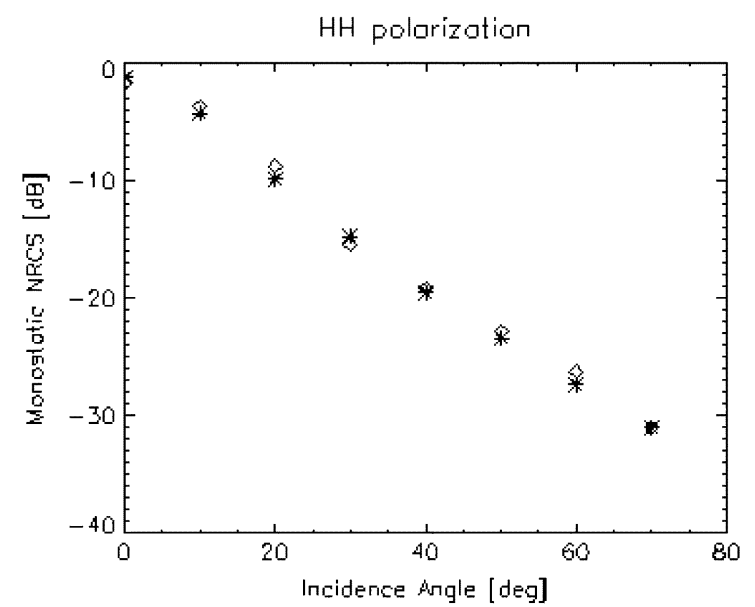

(a)

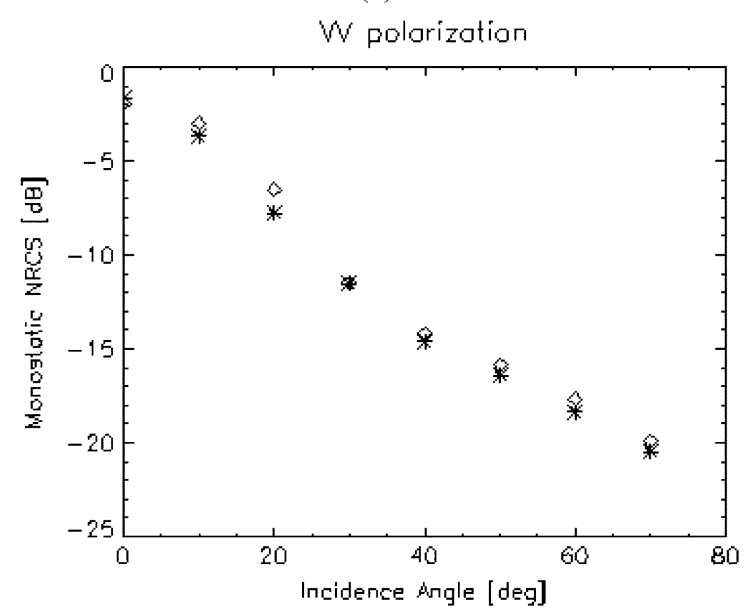

(b)

Fig. 5. Comparison between the X-band (frequency $=10 \mathrm{GHz}$ ) fields scattered by fractal (asterisks) and noisy (boxes) profiles, for (a) $\mathrm{HH}$ and (b) VV polarization.

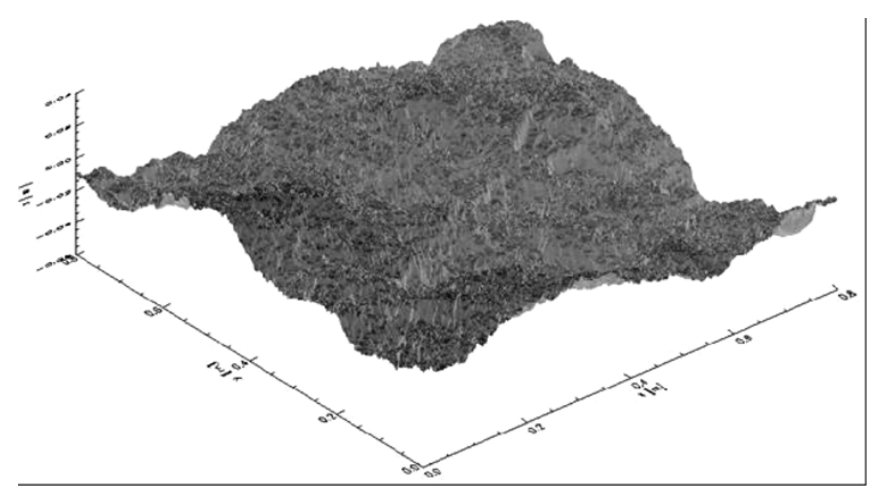

Fig. 6. Digital representation of the built surface (close up).

tion) is $80 \mu \mathrm{m}$, and allows a reliable estimation of the surface fractal parameters of the spatial scales involved in the scattering experiments-see Section II. In Fig. 6, a detail of the digital representation of the constructed surface is presented.
TABLE II

SelF-Similarity BeHAVIOR ANALYSIS. HeRE $\sigma$ STANDS FOR "MEASURED STANDARD DEVIATION OF"

\begin{tabular}{c|c|c|c}
\hline$r$ & $\sigma\{z(r x, r y)\}[\mathrm{m}]$ & $r^{H} \sigma\{z(x, y)\}[\mathrm{m}]$ & Error $(\%)$ \\
\hline 1 & 0.00741 & 0.00741 & 0 \\
\hline$v$ & 0.0089 & 0.0091 & -2.5 \\
\hline$v^{2}$ & 0.0118 & 0.0114 & +3.6 \\
\hline$v^{3}$ & 0.0138 & 0.0141 & -2.0 \\
\hline$v^{4}$ & 0.0179 & 0.0175 & +2.4 \\
\hline$v^{5}$ & 0.0207 & 0.0217 & -4.5 \\
\hline$v^{6}$ & 0.0254 & 0.0269 & -5.3 \\
\hline
\end{tabular}

\section{Fractal Surface Analysis}

In this section, we analyze the characteristics of the surface (see Sections IV and V), with the aim of verifying that the surface complies with the imposed parameters (see Section III). In this way we can provide a fully validated surface for electromagnetic scattering experiments.

The analysis of a fractal surface has been the object of several studies and controversies in literature. In this section, we first verify that the surface has the property of self-affinity (Section IV-A) in order to check that the surface is fractal in the range of scales we imposed. Then we employ two methods to retrieve the fractal parameters $H$ and $s$ : the variogram method (Section IV-B) which analyzes the surface in the spatial domain, and the power spectrum analysis method (Section IV-C) which gives us information on the frequency domain behavior. Compliance of the results of the two methods with the construction operations is discussed in order to provide a full characterization of the surface.

\section{A. Surface Self-Affinity}

As far as the property of self-affinity is concerned, it is known that a surface $z(x, y)$ is self-affine if, for every scaling factor $r$

$$
z(r x, r y) \doteq r^{H} z(x, y)
$$

where $\doteq$ stands for "has the same statistics as."

As the first step in verifying that the surface has this property, we measured the standard deviation of the $z(r x, r y)$ process and we compared it with $r^{H}$ times the standard deviation of the $z(x, y)$ process, for every $r$ value comprised in the range of scales of interest. The results of such an analysis for several surface cuts and for $H=0.7$ are detailed in Table II and show that the surface follows the theoretical approach with an average error lower than 5.3 .

\section{B. Variogram Method}

The variogram $V(\Delta x)$, also referred to as "structure function," is defined as the mean square increment of elevation points placed at distance $\Delta x$

$$
V(\Delta x)=\left\langle(z(x)-z(x+\Delta x))^{2}\right\rangle
$$

For a fractal $\mathrm{fBm}$ surface, the variogram is related to the fractal parameters by the relationship

$$
V(\Delta x)=s^{2} \Delta x^{2 H}
$$




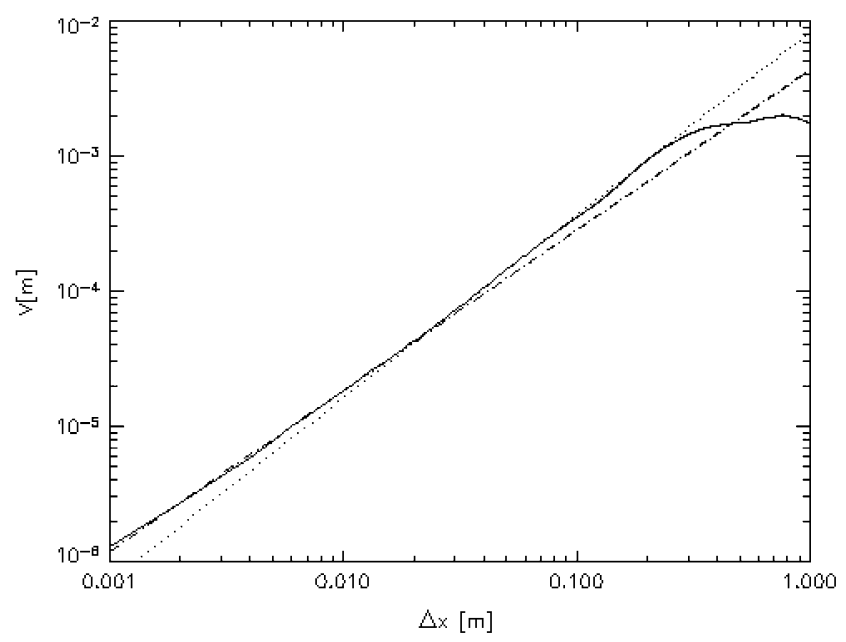

Fig. 7. Variogram behavior: measured data (solid line) match with a straight (dotted) line with slope $2 H=1.36$ (i.e., $H=0.68$ ) at the centimeter scale and with a straight (dashed) line with slope $2 H=1.22$ (i.e., $H=0.61$ ) at the millimeter scale.

whose behavior as a function of the displacement $\Delta x$ in a $\log -\log$ plane, is a linear relationship

$$
\log V(\Delta x)=2 \log s+2 H \log \Delta x
$$

with slope $2 H$, and ordinate intercept $2 \log s$. Accordingly, $H$ and $s$ can be evaluated by performing a linear regression over the log-log plot of measured values of $V(\Delta x)$.

We have evaluated and plotted in Fig. 7 the $V(\Delta x)$ behavior for different $\Delta x$ values on a one-dimensional (1-D) surface cut. We can note that the graph in Fig. 7 can be viewed as the combination of two segments, confirming that the surface can be considered fractal in the whole range of scales of interest, but with different fractal dimension in the centimeter and the millimeter scales. In Fig. 7, in the millimeter scale, the solid line representing the measured values well matches the dashed line, whose slope gives the estimation of the Hurst coefficient $H=$ 0.61 . We verified that the results are independent of the cut direction. This is a first estimation of the wrinkled aluminum foil fractal dimension. At the centimeter scale, the slope changes and the measured data well match the dotted line relative to a fractal surface with the Hurst coefficient $H=0.68$, in agreement with the imposed one, see Section III, Table I. Obtained results turn out to be independent of the direction of the cut, this confirming surface isotropy. If the surface is isotropic, the estimated $H$ value is significant also for the two-dimensional (2-D) case. In addition, we have estimated the intercept of the variogram with the ordinate axis, which is -2.36 for the whole variogram, i.e., $\mathrm{s}=0.066 \mathrm{~m}^{1-\mathrm{H}}$, in agreement with the imposed $s$ value-see Section III.

The variogram analysis provides a global estimate of the fractal dimension, but no information is given about the homogeneity of the surface, hence a local analysis is needed for this information.

The whole surface was divided into rectangular windows, and the fractal dimension inside each window was estimated again with an average variogram method. Several fractal dimension maps can be obtained by this operation, by changing the analyzed window dimensions. In Fig. 8, we show the fractal di-

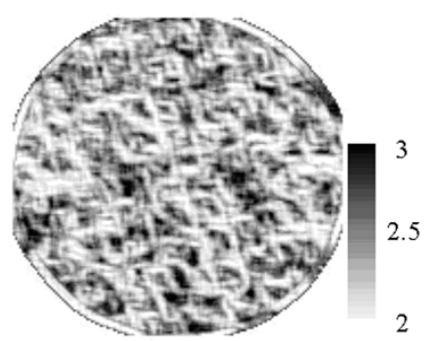

Fig. 8. Fractal dimension map: $D=3-H$.

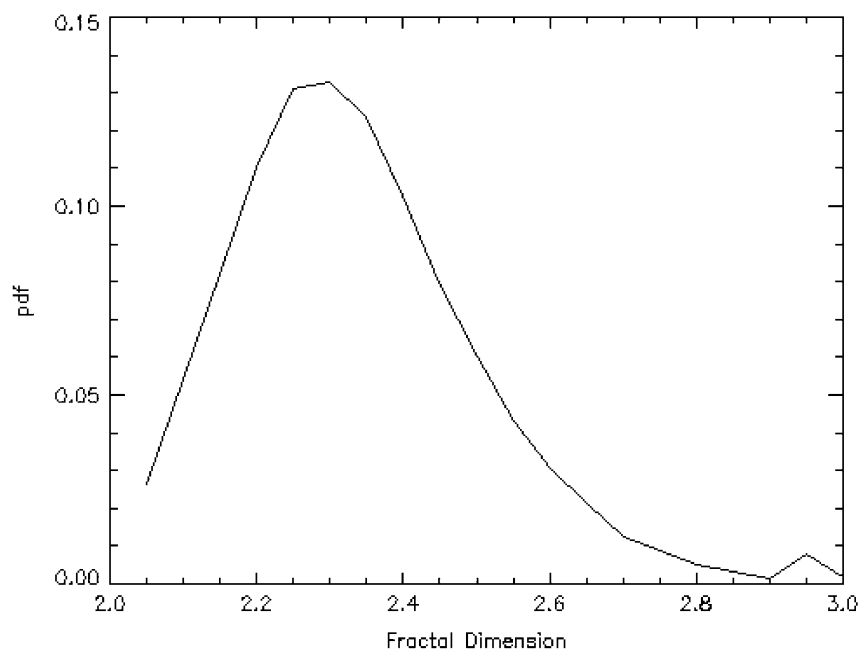

Fig. 9. Probability density function of the fractal dimension.

mension map obtained by the application of this method to our surface with a window dimension of $9 \times 9 \mathrm{~cm}^{2}$.

We evaluated the fractal dimension $D=2.3$, (i.e., $H=0.7$ ) mean value. In Fig. 9, the whole fractal dimension estimated probability density function is plotted. It shows that most of the fractal dimension values are grouped around the 2.30 value, which is the mean value of this distribution and seems to be a significant value for the whole surface fractal dimension. It is in perfect agreement with the fractal dimension value imposed at this scale with the procedure explained in Sections II and III.

\section{Spectral Analysis Method}

Several studies in literature show that natural surfaces have a power law spectrum [see (3.2)]. Hence, spectral analysis is a crucial point of verification as to how well the constructed surface approximates an $\mathrm{fBm}$ process, and, as a consequence, a natural terrain. The techniques to perform this analysis have to take into account the high variance of spectral estimators and spectral leakage problems [23]. In the following, the theoretical issues related to our surface analysis are discussed.

We show, in Fig. 10, the measured power spectrum of the surface at the lowest frequencies (up to $85 \mathrm{~m}^{-1}$ ). The spectrum was obtained as the square modulus of the FFT of the surface, divided by the illuminated area. We can recognize that the surface tones are denser near the lowest and sparser at the highest frequencies, in accordance with the WM definition. Tone directions are randomly placed. The tones appear as peaks in a continuous spectrum, due to the operations performed in building the surface. 


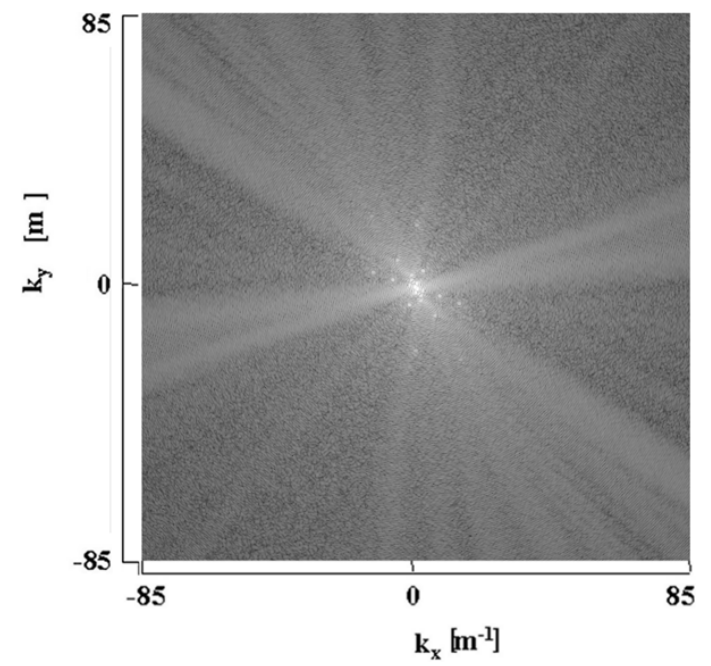

Fig. 10. Two-dimensional Spectral behavior of the built surface.

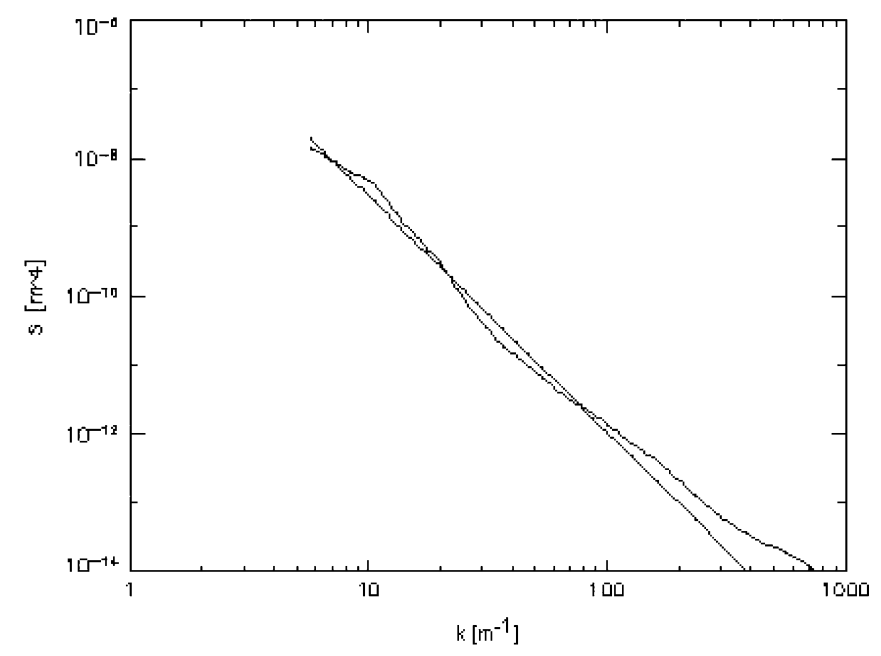

Fig. 11. Spectral decaying compared with the linear fitting curve.

In order to quantitatively estimate if a power law surface spectrum was obtained with the operations performed, we evaluated the maximum value in annular spectral intervals around each tone frequency $\left[k_{0} \nu^{p-0.5}, k_{0} \nu^{p+0.5}\right]$. The spectral decaying is almost linear. We evaluated the mean slope, by means of a linear fit, and we measured a slope $\alpha=3.43$, i.e., an $H=0.71$ value [see (3.3)], in agreement with the imposed fractal dimension. In Fig. 11, we show the measured spectral decaying compared with the line that fits the curve. Obtained results confirm that the surface is a realization of an $\mathrm{fBm}$ process.

\section{Conclusions AND Future Developments}

In this paper, we have presented an innovative method of building a fractal surface. Such a surface can be employed to test electromagnetic scattering methods. The work is intended to provide a solution to the several requirements arising from the wide diffusion of fractal models to describe natural surfaces in electromagnetic scattering theories.
The main constraints that govern all the measurement campaign operations have been accounted for and the solutions chosen have been discussed and summarized in an operational protocol.

The surface parameters were imposed by means of a WM synthesis and their values were set in accordance with the requirements of electromagnetic measurement applications. Computer simulation provided the trace to build the surface. The surface materials were chosen for a simple, quick, and cheap construction procedure and to respect mechanical requirements. In particular, a cardboard topography with a superimposed aluminum foil layer satisfied requirements and ensured the construction of a perfectly conducting surface. The building procedure presented here can be extended to dielectric surface if the penetration depth of the electromagnetic field in the material used is accounted for. In particular, the surface design must be made in such a way that reflections from the surface support will not affect the measurement results.

The surface was measured with an optical instrument in order to obtain a digital elevation map. Finally, the surface was analyzed in order to verify that its parameters complied with the imposed ones. The use of spatial and spectral methods showed that the surface was compliant with the required characteristics and provided a complete description of a surface ready to be used in electromagnetic scattering experiments. This surface is currently being used in an anechoic chamber for scattering measurements aimed at verifying scattering models. Significant and promising results have already been obtained (see [24] and [25]). Complete scattering results will be presented in a paper currently in preparation. These results further encourage the use of $\mathrm{fBm}$ (and its WM approximation) as a model for natural surfaces within electromagnetic scattering evaluation methods.

Note that an $\mathrm{fBm}$ is a Gaussian random process with a power-law spectrum, and some theoretical results in the field of signal processing [27] lead to the conclusion that $\mathrm{fBm}$ is the only possible Gaussian process with a power-law spectrum if the spectral exponent lies in the interval $(2,4)$ for surfaces and $(1,3)$ for profiles. The crucial point is that such a power-law process is nonstationary and has an infinite variance: fortunately, any sensor intrinsically detects a bandlimited version of a natural surface, so that a bandlimited version of $\mathrm{fBm}$ must be used (see Section III and, e.g., [5]). A bandlimited fBm is stationary and has a finite variance [8], [27]; however, its variance and correlation length depend on its lower and upper cutoff frequencies, so they depend also on the sensor, and not only on the natural surface itself. On the contrary, fractal parameters $H$ and $s$ only depend on the surface, and not on the sensor. This is the main reason why it is preferable to use the $\mathrm{fBm}$, defined in terms of fractal parameters ( $H$ and $s$ ), instead of the Gaussian process, defined in terms of classical parameters (variance and correlation length).

\section{REFERENCES}

[1] B. B. Mandelbrot, The Fractal Geometry of Nature. New York: Freeman, 1983. 
[2] K. Falconer, Fractal Geometry: Mathematical Foundations and Applications. New York: Wiley, 1990.

[3] J. S. Feder, Fractals. New York: Plenum, 1988

[4] M. V. Berry and T. M. Blackwell, "Diffractal echoes," J. Phys. A Math. Gen., vol. 14, pp. 3101-3110, 1981.

[5] D. L. Jaggard, "On fractal electrodynamics," in Recent Advances in Electromagnetic Theory, H. N. Kriticos and D. L. Jaggard, Eds. Berlin, Germany: Spinger-Verlag, 1990, pp. 183-223.

[6] A. L. Goldberger and B. J. West, "Fractals in physiology and medicine," Yale J. Biol. Med., vol. 60, no. 5, pp. 421-35, 1987.

[7] B. B. Mandelbrot and V. Ness, "Fractional Brownian motions, fractional noises and applications," SIAM Rev., vol. 10, pp. 422-437, Oct. 1968.

[8] P. Flandrin, "On the spectrum of fractional brownian motions," IEEE Trans. Inf. Theory, vol. 35, no. 1, pp. 197-199, 1989.

[9] G. Franceschetti, A. Iodice, and D. Riccio, "Fractal models for scattering from natural surfaces," in Scattering, R. Pike and P. Sabatier, Eds. London, U.K.: Academic, 2001, pp. 467-485.

[10] P. Beckmann, "Scattering by non-Gaussian surfaces," IEEE Trans. Antennas Propagat., vol. AP-21, no. 2, pp. 169-175, Mar. 1973.

[11] S. C. Wu, M. F. Chen, and A. K. Fung, "Non-Gaussian surface generation," IEEE Trans. Geosci. Remote Sens., vol. 26, no. 6, pp. 885-888, Nov. 1988.

[12] G. Franceschetti, A. Iodice, M. Migliaccio, and D. Riccio, "Scattering from natural rough surfaces modeled by fractional brownian motion two-dimensional processes," IEEE Trans. Antennas Propag., vol. 47, no. 9, pp. 1405-1415, Oct. 1999.

[13] - "Fractals and the small perturbation scattering model," Radio Sci., vol. 34, no. 5, pp. 1043-1054, Sep.-Oct. 1999.

[14] M. V. Berry and Z. V. Lewis, "On the Weierstrass-Mandelbrot fractal function," in Proc. R. Soc. Lond. A, vol. 370, 1980, pp. 459-484.

[15] G. Franceschetti, M. Migliaccio, and D. Riccio, "An electromagnetic fractal-based model for the study of fading," Radio Sci., vol. 31, no. 6, pp. 1749-1759, Nov.-Dec. 1996.

[16] G. Franceschetti, A. Iodice, D. Riccio, and G. Ruello, "Fractal surfaces and electromagnetic extended boundary conditions," IEEE Trans. Geosci. Remote Sens., vol. 40, no. 5, pp. 1018-1031, May 2002.

[17] T. K. Chan, Y. Kuga, A. Ishimaru, and C. T. C. Le, "Experimental studies of bistatic scattering from two-dimensional conducting random rough surfaces," IEEE Trans. Geosci. Remote Sens., vol. 34, no. 3, pp. 674-680, May 1996.

[18] Y. Oh, K. Sarabandi, and F. T. Ulaby, "An empirical model and an inversion technique for radar scattering from bare soil surfaces," IEEE Trans. Geosci. Remote Sens., vol. 30, no. 2, pp. 370-381, Mar. 1992

[19] K. A. O'Donnell and E. R. Mendez, "Experimental study of scattering from characterized random surfaces," J. Opt. Soc. Amer. A, vol. 4, no. 7, Jul. 1987.

[20] S. R. Brown and C. H. Sholz, "Broad band study of the topography of natural rock surfaces," J. Geophys. Res., vol. 90, pp. 12575-12 582, Dec. 1985.

[21] E. Zribi, J. Paillé, V. Ciarletti, O. Taconet, P. Biossard, M. Chapron, and B. Rabin, "Modelization of roughness and microwave scattering of bare soil surfaces based on fractal brownian geometry," in Proc. IGARSS'98, vol. 3, Jul. 1998, pp. 1213-12215.

[22] S. Savaidis, P. Frangos, D. L. Jaggard, and K. Hizanidis, "Scattering from fractally corrugated surfaces with the use of the extended boundary condition method," J. Appl. Phys., vol. 56, pp. 10-21, 1984.

[23] R. T. Austin, A. W. England, and G. H. Wakefield, "Special problems in the estimation of power-law spectra as applied to topographical modeling," IEEE Trans. Geosci. Remote Sens., vol. 32, no. 3, pp. 928-939, Jul. 1994.

[24] G. Franceschetti, A. Iodice, D. Riccio, G. Ruello, P. Blanco, J. Mallorqui, and A. Broquetas, "Electromagnetic scattering from natural surfaces: Validation of a fractal approach," in Proc. URSI Electromagnetic Theory Symposium-EMTS2004, Pisa, Italy, 2004, pp. 1080-1082.

[25] G. Ruello, P. Blanco, A. Iodice, J. J. Mallorqui, D. Riccio, A. Broquetas, and G. Franceschetti, "Electromagnetic scattering measurements on a fractal surface," in Proc. IGARSS, Anchorage, AK, 2004, pp. $425-427$.

[26] M. Davidson, T. Le Toan, F. Mattia, G. Satalino, T. Manninen, and M. Borgeaud, "On the characterization of agricultural soil roughness for radar remote Sens. studies," IEEE Trans. Geosci. Remote Sens., vol. 38, no. 2, pp. 630-640, Mar. 2000.
[27] G. W. Wornell, Signal Processing With Fractals. Upper Saddle River, NJ: Prentice-Hall, 1996

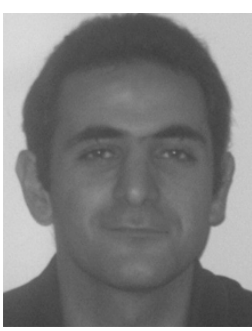

Giuseppe Ruello (S'00-M'04) was born in Naples, Italy, in 1975. He received the Laurea degree (cum laude) in telecommunication engineering in 1999 and the $\mathrm{Ph} . \mathrm{D}$. degree in information engineering in 2003 , both from the University of Naples "Federico II."

In 2000, he received a grant from the University of Naples to be spent at the Department of Electronic and Telecommunication Engineering for research in the field of remote sensing. In 2000, he received a grant from the University of Rome "La Sapienza," Rome, Italy. He was a Visiting Scientist at the Department of Signal Theory and Communications, Universitat Politecnica de Catalunya, Barcelona, Spain, during 2002 and 2004-2005. He is currently a Research Scientist at the Department of Electronic and Telecommunication Engineering of the University of Naples. His main research interests are in the field of synthetic aperture radar (SAR) remote sensing, modeling of electromagnetic scattering from natural surfaces, SAR raw signal simulation, modeling of electromagnetic field propagation in urban environments, and remote sensing techniques for developing countries.

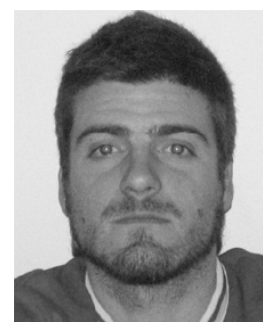

Pablo Blanco-Sánchez was born in Terrassa, Spain, in 1977. He received the B.Sc. degree in physics from Valencia University, Valencia, Spain, in 2001. He is currently pursuing the $\mathrm{Ph}$.D. degree at the Universitat Politècnica de Catalunya (UPC), Barcelona, Spain.

From September to December 2004, he was with the Istituto per il Rilevamento Elettromagnetico dell'Ambiente (IREA), Naples, Italy, in the frame of his $\mathrm{Ph} . \mathrm{D}$. work. His main research interests are in the areas of synthetic aperture radar (SAR) interferometry and SAR differential interferometry

with multiple images.

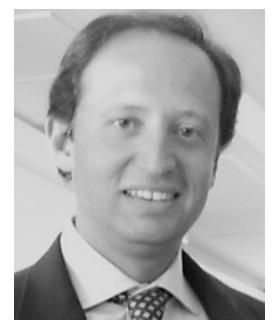

Antonio Iodice (S'97-M'00-SM'04) was born in Naples, Italy, in 1968 . He received the Laurea degree (cum laude) in electronic engineering and the Ph.D. degree in electronic engineering and computer science, both from the University of Naples "Federico II," in 1993 and 1999, respectively.

In 1995, he received a grant from the Italian $\mathrm{Na}$ tional Council of Research (CNR) to be spent at the Istituto di Ricerca per l'Elettromagnetismo e i Componenti Elettronici (IRECE), Naples, for research in the field of remote sensing. He was with Telespazio S.p.A., Rome, Italy, from 1999 to 2000. From 2000 to 2004, he was a Research Scientist at the Department of Electronic and Telecommunication Engineering, University of Naples "Federico II," where he is currently a Professor of Electromagnetics. His main research interests are in the field of microwave remote sensing and electromagnetics: modeling of electromagnetic scattering from natural surfaces and urban areas, simulation and processing of synthetic aperture radar (SAR) signals, and SAR interferometry. He is the author or coauthor of about 100 papers published in refereed journals or in proceedings of international and national conferences. 


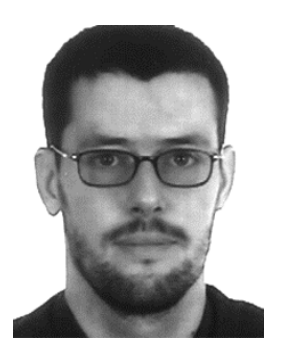

Jordi J. Mallorquí (S'90-M'96) was born in Tarragona, Spain, in 1966. He received the Ing. degree in telecommunication engineering in 1990 and the Dr. Ing. degree in telecommunications engineering in 1995 for research on microwave tomography for biomedical applications, both from the Universitat Politècnica de Catalunya (UPC), Barcelona, Spain.

In 1991, he joined the Department of Signal Theory and Communications as Ph.D. student. In 1993, he became an Assistant Professor, and since 1997 has been an Associate Professor at the Telecommunications Engineering School, UPC, Barcelona. His teaching activities involve microwaves, radionavigation systems, and remote sensing. He spent a sabbatical year at the Jet Propulsion Laboratory, Pasadena, CA, in 1999, working on interferometric airborne synthetic aperture radar (SAR) calibration algorithms. He is currently working on the application of SAR interferometry to terrain deformation monitoring with orbital and airborne data, vessel detection, and classification from SAR images and three-dimensional electromagnetic simulation of SAR systems. He is also collaborating in the design and construction of a ground-based SAR interferometer for landslide control. Finally, he is now developing the hardware and software of a bistatic parasitic SAR for interferometric applications using ERS and ENVISAT as sensors of opportunity. He has published more than 80 papers on microwave tomography, electromagnetic numerical simulation, SAR processing, and interferometry and differential interferometry in referred journals and international symposia.

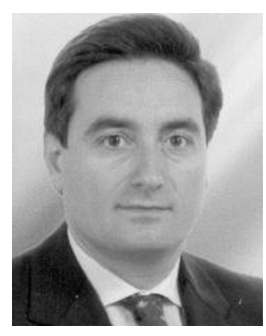

Daniele Riccio (M'91-SM'99) was born in Naples, Italy, in 1962. He received the Laurea degree (cum laude) in electronic engineering from the University of Naples "Federico II" in 1989.

$\mathrm{He}$ is a Professor of Electromagnetics and Remote Sensing at the University of Naples "Federico II." He was Research Scientist at the Istituto di Ricerca sull'Elettromagnetismo e i Componenti Elettronici (IRECE) of the Italian National Council of Research (CNR) and at the Department of Electronic and Telecommunication Engineering, University of Naples "Federico II." In 1994 and 1995, he was also Guest Scientist at the DLR High-Frequency Institute, Munich, Germany. His research activities have resulted in 150 papers published in the fields of microwave remote sensing, synthetic aperture radar simulation, modeling and information retrieval for land, oceanic, and urban scenes, as well as in the application of fractal geometry to electromagnetic scattering from natural surfaces and to remote sensing.

Professor Riccio has won several fellowships from private and public companies (SIP, Selenia, CNR, CORISTA, CRATI) for research in the remote sensing field.

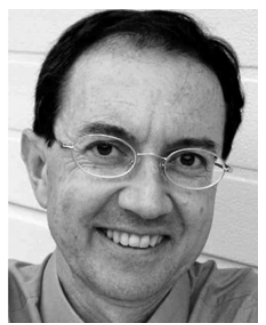

Antoni Broquetas ( ' $\left.^{\prime} 84-\mathrm{M}^{\prime} 90\right)$ was born in Barcelona, Spain, in 1959. He received the Ing. degree in telecommunication engineering from the Universitat Politècnica de Catalunya (UPC), Barcelona, Spain, in 1985 and the Dr. Ing. degree in telecommunications engineering in 1989 for his work on microwave tomography in the UPC.

In 1986, he was a Research Assistant at Portsmouth Polytechnic, Portsmouth, U.K., involved in propagation studies. In 1987, he joined the Department of Signal Theory and Communications, School of Telecommunication Engineering, UPC. From 1998 to 2002, he was Subdirector of Research at the Institute of Geomatics, Barcelona. Since 1999, he has been a Full Professor at the UPC, involved in research on radar imaging and remote sensing. Since 2003, he has been the Director of the Signal Theory and Communications Department, UPC. He has published more than 150 papers on microwave tomography, radar, ISAR and SAR systems, SAR processing, and interferometry.

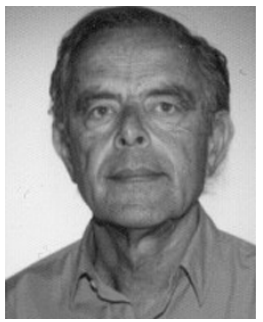

Giorgio Franceschetti (M'62-SM'85-F'88-LF'01) was born in Italy. He was a Full Professor of Electromagnetic Wave Theory at the University of Naples, Naples, Italy, since 1968. He was a Visiting Professor at the University of Illinois, Urbana-Champaign, in 1976 and 1977, and at the University of California at Los Angeles (UCLA) in 1980 and 1982. He was a Research Associate at Caltech, Pasadena, CA, in 1981 and 1983, a Visiting Professor at National Somali University in 1984, and a Visiting Professor at the University of Santiago de Compostela, Spain, in 1995. He was a Fulbright Scholar at Caltech in 1973. He has lectured in several summer schools in China, the U.K., Holland, Italy, Spain, Sweden, and the U.S. He was Director of IRECE, a Research Institute of the Italian National Council of Research (CNR) and member of the board of the Italian Space Agency (ASI). $\mathrm{He}$ is currently also an Adjunct Professor at UCLA, and a Distinguished Visiting Scientist at the Jet Propulsion Laboratory (JPL), Pasadena, CA. He has published several books and more than 140 refereed papers in the field of applied electromagnetics (reflector antennas, transient phenomena, shielding, nonlinear propagation, and scattering) and, more recently, in the field of SAR data processing and simulation.

Prof. Franceschetti has received several national and international awards. 